\title{
MJN \\ MEDICATION KNOWLEDGE AND ADHERENCE AMONG TYPE II DIABETES MELLITUS PATIENTS: A CROSS SECTIONAL STUDY
}

\author{
Omed Saadallah Al-Amedy ${ }^{1 *}$, Tang Li Yoong², Gulistan Ahmed Saido³ \\ ${ }^{1}$ Nursing Department, College of Nursing, University of Duhok(UoD),Kurdistan Region, Duhok \\ City, Iraq \\ ${ }^{2}$ Department of Nursing Science, Faculty of Medicine, University of Malaya, Malaysia \\ ${ }^{3}$ Department of Biophysics, College of Medicine, University of Duhok(UoD), Kurdistan Region, \\ Duhok, Iraq
}

*Corresponding Author's Email:omed.saadallah@uod.ac

\begin{abstract}
Diabetes is a chronic complicated disease. For that reason, self-care management and education are necessary to control blood glucose levels todecrease mortality and morbidity rate, the risk of complications and adverse medication related events. Lack of knowledge about a diabetic medication and non-adherence contribute to poor glycemic control among diabetes patients. Therefore, this study aims to assess relationship between medication knowledge and medication adherence among T2DM. A cross sectional study was carried out in outpatient's diabetic clinic in University Malaya Medical Centrevia recruiting 250 participants. A set of questionnaires from earlierresearch were used to collect the data. Descriptive and inferential statistical analysis is used to analyze the data. The inclusive results indicated that, the mean age of the participants was $57.90 \pm 13.22$ within 56years. More than half of patients $(52.5 \%)$ had poor medication knowledge, and $47.5 \%$ had good knowledge. In terms of medication adherence, most of the participants $(87.0 \%)$ had medium medication adherence; whereas, only $1.3 \%$ showed high medication adherence. However, there was no correlation between medication knowledge and medication adherence $(p=0.743)$. The chi-square results indicated that medication knowledge was associated significantly with gender, educational level, employment status and monthly income ( $p$ value $<0.05)$. In conclusion it can be said that this study provides insight for healthcare professional about medication knowledge and adherence which are important for managing diabetes. Awareness of these factors will allow them to be more effective about medication counseling so that patients can become self-responsible and realize the benefits of prescribed therapies.
\end{abstract}

\section{Keywords: Medication Knowledge, Medication Adherence, Type II Diabetes Mellitus}

\section{INTRODUCTION}

Diabetes Mellitus is a worldwide health problem. The number of patients with hyperglycemia is increasing. This is evident from the findings of a sequence of wide-reaching estimates of current and predicted in-future prevalence of diabetes according to the latest update in 2004 (Shaw, Sicree, \& Zimmet, 2010). Type II Diabetes Mellitus Patients (T2DM) consist of $90-95 \%$ of all the cases of DM that happens in middle age and influence the existence of the individuals (Chew et al., 2012). Having diabetes is one of the most common endocrine disorders. It is estimated that more than 300 million will subsequently suffer from this disease by 2025, (Nesto, 2004). This developing trend is due to severalfeatures such as population growing, aging, urbanization and increasing prevalence of obesity and physical inactivity (Danaei et al., 2011). The occurrence of diabetes in Malaysia continues to increase at an alarming rate from $1-2 \%$ in the 1960 s and 
1970 s, $6.3 \%$ in 1986 (NHMS I), $8.3 \%$ in 1996 to $14.9 \%$ in 2006 (NHMS III). According to the Malaysian Burden of Disease and Injury Study, it was estimated that for the year 2000, there were 2,261 deaths attributed to diabetes (857 men and 1404 women) (Chew et al., 2012; Kosek, Bern \& Guerrant, 2003).

However, to gain glycemic control, researches advocated that patients must undergo certain oral treatments and/or insulin along with adhering to careful diet plans, exercise regimes and monitor their blood glucose regularly. However, with the development of the disease, patients must take extra medications to manage and prevent microvascular and macrovascular complications. In other words, glycemic control is strictly dependent on the patients' capabilities of selfmanagement and care. Due to the complex nature of the disease, treatment for T2DM can be very complex. So, handling diabetes successfully is challenging amongst diabetic patients. On the other hand, studies that evaluated diabetes-related knowledge at one point of a time have shown a positive correlation with blood glucose control. Hence, those DM patients with little literacy and low knowledge potency might face distress in learning self-care skills on behalf of glycemic regulator, which possibly will be affected by illiteracy, as well as reduced visual and hearing status as an effect of aging progression. Moreover, previous researches on diabetes emphasized that poor glycemic control is associated with non-adherence to prescribed medications (Omar \& San, 2014).

World Health Organization (WHO) defines adherence as "the extent to which a person's medicationtaking behavior, following a diet, and/or executing lifestyle changes, corresponds with the agreed recommendations from a health-care provider". Positive health outcomes and lower mortality among patients with diabetes have been associated with good adherence compared to those patients with poor adherence (O'Kane et al., 2008). However, nonadherence to the prescribed medication leads to poor diabetic control and infection, which are the causes of frequent hospital admissions and readmissions (Vlasnik, Aliotta \& DeLor, 2005). Poor adherence not only decreases the whole efficacy of medications but is also associated with increased complications and higher mortality rates. Furthermore, it has been shown that patients who maintain a high rate of medication compliance have significantly reduced rates of hospitalization, thus reducing the cost in healthcare (Sokol et al., 2005). Hence, medication knowledge and adherence are the most significant variables disturbing the management of diabetes. The main aims of this study are to assess the relationship between the medication knowledge and adherence among T2DM patients.

\section{RESEARCH METHODOLOGY}

\section{Study Design, Setting, Participants and Procedure}

A descriptive cross-sectional study was carried out to determine the relationship between medication knowledge and adherence of medication among T2DM patients. Raosoft online software, accessible in website http://www.raosoft.com/samplesize.html; was used by direct estimated real sample size of 215 patients. The researcher distributed a set of 250 questionnaires among. The researcher distributed a set of 250 questionnaires among the participants, while only 235 participants participated in the research. In order to reduce inaccurate results and increase the reliability of the study, only $(0.12 \%)$ sets were excluded from the study because the information given were incomplete. Finally, 223 participants were included in the analysis.

The inclusion criteria of the study were; 1) patients with age $>18$ years, 2) patient diagnosed $>6$ months with T2DM, 3) able to understand either English or Bahasa Malaysia language, or 4) patient free from any psychiatric disorders or diseases. Patients with diabetes mellitus complications, and unable to sign informed consent were excluded from the study.

\section{Research Instruments-Questionnaire}

Data were collected using close-ended questions and self-administered questionnaire by the researcher. It consisted of 39 items divided into 3 sections. Section (A) consisted of 13 items correlated to socio-demographic characteristics of the participants. Section (B) served to determine medication knowledge level among T2DM patients, that consisted of 18 items in the form of 5-point Likert scale ranged as $(1=$ strongly agree, $2=$ agree, $3=$ neutral, $4=$ disagree and $5=$ strongly disagree) which was adopted and modified from Burge, Albright \& RRNeST, 2005 after taking permission from the author. Point Zero was given for wrong answers in the first 3 statement (strongly agree, agree, and neutral). Whileone was given for correct answers of $4^{\text {th }}$ and $5^{\text {th }}$ statement (disagree and strongly disagree). Therefore, the total sum of possible scores in this scale ranged from $(0-18)$. 
Further, score is divided into two groups, $(<9)$ considered as poor medication knowledge and $(>9)$ considered as good medication knowledge. MMAS-8 Morisky scale used to determine the medication adherence level among T2DM patients. Therefore, the total sum of possible scores range from $(0-8)$ and divided into 3 groups, $(<6)$ considered as low medication adherence, $(6-<8)$ consider as medium medication adherence and $(=8)$ is considered high medication adherence. The questionnaires were in English and Bahasa Malaysia Language, so that patient has a choice to answer either in English or in Bahasa Malaysia Language.

\section{Data Collection and Ethical Consideration}

This study was approved by the Medical Ethics Committee MEC in University Malaya Medical Centre (UMMC). A copy of ethical approval was sent to head of nursing in (UMMC) and outpatient's diabetes clinic. Data collection conducted by self-administer from $5^{\text {th }}$ March until $2^{\text {nd }}$ April 2015.

\section{Statistical Analysis}

Data were entered, analyzed and coded into a database using SPSS software ver.21 to maintain confidentiality for all patients. The collective score of the instruments was entered as a continuous data variable to measure and used to analyze performance according to the research questions underlying the study. A descriptive statistics analysis was used to describe socio-demographic and clinical characteristics of the respondent such as frequencies and percentage was used for the categorical variables, whereas the mean and std. deviation were used to calculate the continuous variables. An Inferential statistical analysis was used to determine the association between sociodemographic characteristics, medication knowledge and adherence of medication.

\section{RESULTS}

\section{Socio-Demographic Characteristics}

The normal distribution test done for 223 participants and the results showed the data are normally distributed for medication knowledge and medication adherence variable were lies within $\pm 1(-1.96-1.96)$ of skewness and kurtosis respectively. As shown in Table 1, the mean age of the participants was $57.90 \pm 13.22$ $(\mathrm{n}=223)$ with minimum and maximum age being 31 and 87 respectively. About $35.4 \%$ of the participants were between age groups $50-64$. More than half (51.6\%) were female while $48.4 \%$ were male. About $47.1 \%$ was Malay followed by Indian and Chinese. Furthermore, $50.2 \%$ had secondary schooling, $29.1 \%$ were retired and $50.2 \%$ of the participants had a monthly income of 1000 -3000 RM. Moreover, 76.7\% had Family history of DM, while $42.2 \%$ of the participants had been diagnosed with DM for $10-19$ years. $57.0 \%$ of the participants received DMinformation from Doctors and 39.5\% received information from Nurses. Finally, the BMI of the sample was $25.52 \pm 3.0433$ with $50.2 \%$ of the participants having Ideal BMI.

Table 1: Respondents Characteristics at the Baseline $(n=223)$

\begin{tabular}{|c|c|c|}
\hline Variables & $\mathbf{n}$ & (\%) \\
\hline \multicolumn{3}{|l|}{ Age (Year) } \\
\hline$<49$ & 70 & (31.4) \\
\hline $50-64$ & 79 & $(35.4)$ \\
\hline$\geq 65$ & 74 & $(33.2)$ \\
\hline \multicolumn{3}{|l|}{ Gender } \\
\hline Male & 70 & $(31.4)$ \\
\hline Female & 79 & $(35.4)$ \\
\hline \multicolumn{3}{|l|}{ Ethnicity } \\
\hline Malay & 105 & $(47.1)$ \\
\hline Chinese & 47 & $(21.1)$ \\
\hline Indian & 64 & (28.7) \\
\hline Others & 7 & $(3.1)$ \\
\hline \multicolumn{3}{|l|}{ Educational Level } \\
\hline Not Educated & 16 & $(7.2)$ \\
\hline Primary School & 25 & $(11.2)$ \\
\hline Secondary School & 112 & $(50.2)$ \\
\hline $\begin{array}{l}\text { Tertiary (University - } \\
\text { College) }\end{array}$ & 70 & (31.4) \\
\hline \multicolumn{3}{|l|}{ Marital Status } \\
\hline Single & 15 & (6.7) \\
\hline Married & 166 & $(74.4)$ \\
\hline Separated & 4 & $(1.8)$ \\
\hline Divorced & 6 & $(2.7)$ \\
\hline
\end{tabular}




\begin{tabular}{|c|c|c|}
\hline Widowed & 32 & $(14.3)$ \\
\hline \multicolumn{3}{|l|}{ Employment Status } \\
\hline Unemployed & 48 & $(21.5)$ \\
\hline Self-employed & 17 & $(7.6)$ \\
\hline Government & 34 & $(15.2)$ \\
\hline Private Sector & 59 & $(26.5)$ \\
\hline Retired & 65 & (29.1) \\
\hline \multicolumn{3}{|l|}{ Monthly Income (MYR) } \\
\hline$<1000$ & 54 & $(24.2)$ \\
\hline $1000-3000$ & 112 & $(50.2)$ \\
\hline$>=3000$ & 57 & $(25.6)$ \\
\hline \multicolumn{3}{|l|}{ Family History of DM } \\
\hline Yes & 171 & $(76.7)$ \\
\hline No & 52 & $(23.3)$ \\
\hline \multicolumn{3}{|l|}{$\begin{array}{l}\text { Duration of DM } \\
\text { Diseases (Year) }\end{array}$} \\
\hline$<9$ & 64 & $(28.7)$ \\
\hline $10-19$ & 94 & $(42.2)$ \\
\hline$>=20$ & 65 & $(29.1)$ \\
\hline \multicolumn{3}{|l|}{ Type of DM Medication } \\
\hline Oral Hypoglycaemic Agent & 54 & $(24.2)$ \\
\hline Insulin only & 65 & $(29.1)$ \\
\hline OHA \& insulin & 104 & $(46.6)$ \\
\hline \multicolumn{3}{|l|}{$\begin{array}{l}\text { Number of Oral DM } \\
\text { Medication }\end{array}$} \\
\hline 1 & 25 & $(11.2)$ \\
\hline $2-3$ & 55 & $(24.7)$ \\
\hline$>=3$ & 143 & $(64.1)$ \\
\hline \multicolumn{3}{|l|}{ Insulin Injection Per Day } \\
\hline Once a day & 34 & $(15.2)$ \\
\hline Twice a day & 59 & $(26.5)$ \\
\hline 2 - 3 Times a day & 110 & $(49.3)$ \\
\hline $4 \&$ above & 20 & $(9.0)$ \\
\hline
\end{tabular}

\begin{tabular}{|c|c|c|}
\hline Recent HbA1c Value (\%) & & \\
\hline$<6.5$ & 55 & $(24.7)$ \\
\hline$>=6.5$ & 168 & $(75.3)$ \\
\hline \multicolumn{2}{|c|}{} \\
\hline$<18.5$ Under weight & 25 & $(11.2)$ \\
\hline 18.5 - 24.9 Ideal & 112 & $(50.2)$ \\
\hline 25.0 - 29.9 Over weight & 69 & $(30.9)$ \\
\hline$>30$ Obese & 17 & $(7.6)$ \\
\hline
\end{tabular}

In order to determine the relationship between medication knowledge and medication adherence, Pearson correlation coefficient test was used. The results showed in Table 2 indicated the $p$-value 0.743 which is $>0.05$, thus there is no association between medication knowledge and medication adherence among T2DM patients. However, the total number of participants with poor medication knowledge $(n=117)$ was greater than the total number of participants with good medication knowledge $(n=106)$. In terms, the majority of the participants had medium medication adherence $(6-<8)(n=194)$ followed by low medication adherence $(<6)$. Only $(n=3)$ out of 223 participants had high medication adherence.

Table 2: Association between Medication Knowledge and Medication Adherence $(n=223)$

\begin{tabular}{|c|c|c|c|c|c|c|c|c|}
\hline \multirow{3}{*}{ Variables } & \multicolumn{6}{|c|}{ Edication Adherence (MMAS-8) } & \multirow{3}{*}{$\begin{array}{c}P \\
\text { value }\end{array}$} & \multirow{3}{*}{$\begin{array}{l}\text { Total } \\
(100)\end{array}$} \\
\hline & \multicolumn{2}{|c|}{$<6$ Low } & \multicolumn{2}{|c|}{$6-<8$ Medium } & \multicolumn{2}{|c|}{$>=8$ High } & & \\
\hline & $\mathrm{n}$ & $(\%)$ & $\mathrm{n}$ & $(\%)$ & $\mathrm{n}$ & $(\%)$ & & \\
\hline $\begin{array}{l}\text { Poor Medication } \\
\text { Knowledge }\end{array}$ & 15 & $(12.8)$ & 100 & $(85.5)$ & 2 & $(1.7)$ & & 117 \\
\hline $\begin{array}{l}\text { Good Medication } \\
\text { Knowledge }\end{array}$ & 11 & $(10.4)$ & 94 & $(88.7)$ & 1 & $(0.9)$ & 0.743 & 106 \\
\hline Total & 26 & $(11.7)$ & 194 & $(87.0)$ & 3 & $(1.3)$ & & 223 \\
\hline
\end{tabular}




\section{Association between Socio-Demographic and Medication Knowledge among T2DM}

In order to determine the association between sociodemographic and medication knowledge, Chi-square test was used. As showed in Table 3, the majority of participants who had poor medication knowledge were in age groups 50-64. The gender, educational level, employment status, and monthly income were found to be significant factors to medication knowledge ( $P$-value $<0.05)$. Lastly, in spite of family history of DM, it was not found to be a significant factor in this study. While the participant with no family history of DM were poorly knowledgeable about their medication (63.5\%) in comparison to the participants who had a family history of DM (50.9\%), who had good medication knowledge.

\section{Table 3: Association between Socio-Demographic and Medication Knowledge}

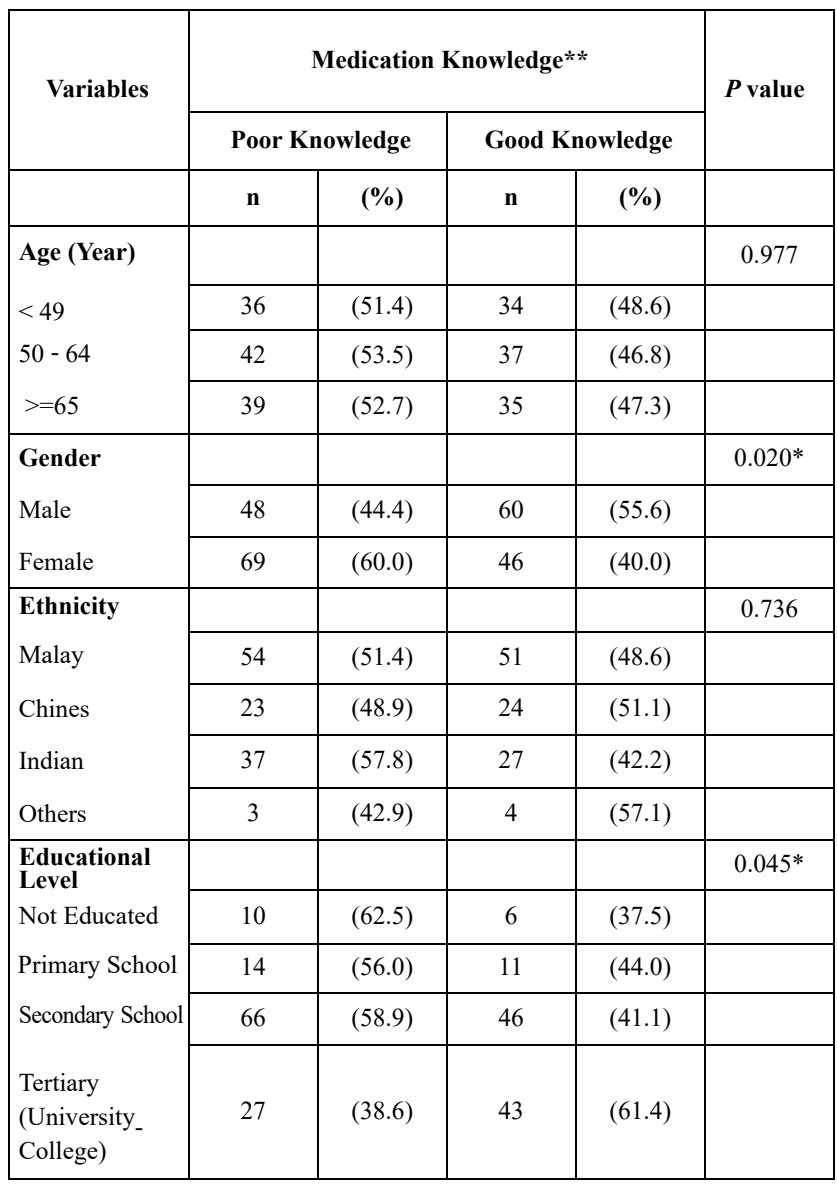

\begin{tabular}{|c|c|c|c|c|c|}
\hline \multirow{2}{*}{$\begin{array}{l}\text { Employment } \\
\text { status } \\
\text { Unemployed }\end{array}$} & & & & & $0.028 *$ \\
\hline & 26 & $(54.2)$ & 22 & $(45.8)$ & \\
\hline Self-employed & 6 & (35.3) & 11 & $(64.7)$ & \\
\hline Government & 14 & $(41.2)$ & 20 & $(58.8)$ & \\
\hline $\begin{array}{l}\text { Private } \\
\text { Sector }\end{array}$ & 27 & (45.8) & 32 & $(54.2)$ & \\
\hline Retired & 44 & $(67.7)$ & 21 & (32.3) & \\
\hline $\begin{array}{l}\text { Monthly } \\
\text { income (RM) }\end{array}$ & & & & & $0.055^{*}$ \\
\hline$<1000$ & 34 & $(63.0)$ & 20 & $(37.0)$ & \\
\hline $1000-3000$ & 60 & (53.6) & 52 & (46.4) & \\
\hline$\geq 3000$ & 23 & $(40.4)$ & 34 & $(59.6)$ & \\
\hline $\begin{array}{l}\text { Family } \\
\text { history of } \\
\text { DM }\end{array}$ & & & & & 0.070 \\
\hline Yes & 84 & $(49.1)$ & 87 & $(50.9)$ & \\
\hline No & 33 & $(63.5)$ & 19 & $(36.5)$ & \\
\hline
\end{tabular}

RM= Malaysian Ringgit; DM= Diabetes Mellitus

*Significant level $p<0.05$

**Chi-square test

\section{Association between Socio-Demographic and Medication Adherence among T2DM}

In order to determine the association between sociodemographic and medication adherence, chi-square test was used as shown in table 4 . The mean and standard deviation of medication adherence was $1.90 \pm 0.34$ respectively. More than half of the participants in age groups of 65 years had high medication adherence. The majority of gender was female $(87.0 \%)$ with medium medication adherence and $89.4 \%$ of them were Chinese patients. Most of the participants $(93.8 \%)$ who are not educated had medium medication adherence with $(94.1 \%)$ who are self-employed. Furthermore, the results indicated that monthly income is not a significant factor for medication adherence. Conversely, the majority of the participants $(91.2 \%)$ with monthly income $>3000$ RM had medium medication adherence. This was true with the patients who had family history of DM. 
Table 4: Association between Socio-Demographic and Medication Adherence

\begin{tabular}{|c|c|c|c|c|c|c|c|}
\hline \multirow{3}{*}{ Variables } & \multicolumn{6}{|c|}{ Medication Adherence ** } & \multirow{3}{*}{$P$ value } \\
\hline & \multicolumn{2}{|c|}{$<6$ Low } & \multicolumn{2}{|c|}{$6-<8$ Medium } & \multicolumn{2}{|c|}{$>=8$} & \\
\hline & $\mathbf{n}$ & (\%) & $\mathbf{n}$ & $(\%)$ & $\mathbf{n}$ & $(\%)$ & \\
\hline \multirow{4}{*}{$\begin{array}{l}\text { Age (Year) } \\
<49 \\
50-64 \\
\geq 65\end{array}$} & & & & & & & 0.666 \\
\hline & 7 & $(10.0)$ & 62 & $(88.6)$ & 1 & (1.4) & \\
\hline & 10 & $(12.7)$ & 69 & $(87.3)$ & 0 & $(0.0)$ & \\
\hline & 9 & $(12.2)$ & 63 & $(85.1)$ & 2 & (2.7) & \\
\hline \multirow{3}{*}{$\begin{array}{l}\text { Gender } \\
\text { Male } \\
\text { Female }\end{array}$} & & & & & & & 0.861 \\
\hline & 13 & $(12.0)$ & 94 & $(87.0)$ & 1 & $(0.9)$ & \\
\hline & 13 & $(11.3)$ & 100 & $(87.0)$ & 2 & $(1.7)$ & \\
\hline \multirow{5}{*}{$\begin{array}{l}\text { Ethnicity } \\
\text { Malay } \\
\text { Chinese } \\
\text { Indian } \\
\text { Others }\end{array}$} & & & & & & & 0.880 \\
\hline & 12 & $(11.4)$ & 91 & $(86.7)$ & 2 & $(1.9)$ & \\
\hline & 5 & $(10.6)$ & 42 & $(89.4)$ & 0 & $(0.0)$ & \\
\hline & 9 & $(14.1)$ & 54 & $(84.4)$ & 1 & (1.6) & \\
\hline & 0 & $(0.0)$ & 7 & $(100.0)$ & 0 & $(0.0)$ & \\
\hline \multirow{5}{*}{$\begin{array}{l}\text { Educational Level } \\
\text { Not Educated } \\
\text { Primary School } \\
\text { Secondary School } \\
\text { Tertiary (University-College) }\end{array}$} & & & & & & & 0.419 \\
\hline & 1 & (6.3) & 15 & (93.8) & 0 & $(0.0)$ & \\
\hline & 2 & $(8.0)$ & 23 & $(92.0)$ & 0 & $(0.0)$ & \\
\hline & 17 & $(15.2)$ & 92 & $(82.1)$ & 3 & (2.7) & \\
\hline & 6 & $(8.6)$ & 64 & (91.4) & 0 & $(0.0)$ & \\
\hline \multirow{6}{*}{$\begin{array}{l}\text { Employment Status } \\
\text { Unemployed } \\
\text { Self-employed } \\
\text { Government } \\
\text { Private Sector } \\
\text { Retired }\end{array}$} & & & & & & & 0.900 \\
\hline & 7 & $(14.6)$ & 41 & $(85.4)$ & 0 & $(0.0)$ & \\
\hline & 1 & (5.9) & 16 & $(94.1)$ & 0 & $(0.0)$ & \\
\hline & 5 & (14.7) & 28 & $(82.4)$ & 1 & (2.9) & \\
\hline & 5 & $(8.5)$ & 53 & $(89.8)$ & 1 & (1.7) & \\
\hline & 8 & $(12.3)$ & 56 & $(86.2)$ & 1 & $(1.5)$ & \\
\hline \multirow{4}{*}{$\begin{array}{l}\text { Monthly Income (RM) } \\
<1000 \\
1000-3000 \\
\geq 3000\end{array}$} & & & & & & & 0.209 \\
\hline & 4 & (7.4) & 48 & $(88.9)$ & 2 & (3.7) & \\
\hline & 17 & $(15.2)$ & 94 & $(83.9)$ & 1 & $(0.9)$ & \\
\hline & 5 & $(8.8)$ & 52 & $(91.2)$ & 0 & $(0.0)$ & \\
\hline \multirow{3}{*}{$\begin{array}{l}\text { Family History of DM } \\
\text { Yes } \\
\text { No }\end{array}$} & & & & & & & 0.571 \\
\hline & 18 & $(10.5)$ & 151 & $(88.3)$ & 2 & $(1.2)$ & \\
\hline & 8 & $(15.4)$ & 43 & $(82.7)$ & 1 & (1.9) & \\
\hline
\end{tabular}

RM= Malaysian Ringgit; $D M=$ Diabetes Mellitus

*Significant level $p<0.05$

**Chi-square test

\section{DISCUSSION}

Diabetes is a common chronic disease. Self-care managements are obligatory to control blood glucose levels. Therefore, this study aims to assess medication knowledge and adherence as well as determine the relationship between these two variables among 223T2DM patients. The results showed that the mean age was $57.90 \pm 13.22(\mathrm{n}=223)$. However, Letchuman et al., (2010) also indicated that T2DM is more widespread among patients with age groups 50-65 and this finding is further supported by Omar \& San (2014) who established that T2DM is projecting in the middle of early elderly rather than the old aging. In terms of gender, the results revealed that T2DM is more common among female patients than male. This finding is agreed with Hernandez et al., (2012) who did a study in southeastern United States with 378 adults with T2DM.

In terms of medication knowledge, the greater number of participants with poor medication knowledge were in age groups $>65$, while $48.6 \%$ of the participants who had good medication knowledge were in age $<49$. In this study, $60.0 \%$ were female participants having poorer medication knowledge which is also supported by Kosek, Bern \& Guerrant, (2003). In addition, Mosher 
et al., (2012) tested that patients with lower literacy have poorer medication knowledge. In the same context Burge, Albright \& RRNeST, (2005) did a study to examine reasons related to medication knowledge and medication adherence in family medicine patients with chronic condition. Their finding indicated that patient education level was associated with better medication knowledge which is consistent to the findings of this study that indicated that educational level was also found to influence medication knowledge score. Good medication knowledge score was only reached by participants having advanced educational level. Therefore, appropriate strategies must be edged to help the poorly educated patients, such as giving them leaflets that are easy to read, professionals who are competent to carry information successfully via verbal communication and recognizing difficulties in communication. Furthermore, the duration of DM plays a significant role in managing of diabetes. The findings of Sajith et al., (2014) study showed that $43.81 \%$ patients had a diabetic history of $1-5$ years and $76.19 \%$ of patients did not have a family history of DM, while in this study the greater number of participants $(63.5 \%)$ had no family history of DM. In short, the findings on medication knowledge indicated that medication knowledge was associated significantly with gender, educational level, employment status and monthly income, due to $p$-value $<0.05$. These findings are further supported by previous studies in T2DM (A1Qazaz et al., 2011; Jimmy et al., 2014; Mastura et al., 2011).

Furthermore, medication adherence has a greater influence on outcomes in chronic diseases. However, as many chronic disease patients often do not experience noticeable symptoms, following treatment recommendations can be difficult. Satisfaction by means of treatment and quality of life are connected by employment status and higher income. People with lower education level and unemployment persons have in general lower satisfaction with life and are less satisfied with diabetes treatment as well as having worse metabolic control (Sajith et al., 2014). Findings of this study indicated that $93.8 \%$ of the non-educated participants and government employees were in medium medication adherence. This finding was further supported by Jimmy et al., (2014). Research regarding medication adherence in T2DM emphasized that patients with a longer history of the disease would be keener to adhere to their medications since complications would have developed, or that they would have a greater awareness and fear of developing them. In short, the results of this study indicated that medication adherence is not associated with sociodemographic features such as age, gender, educational level, ethnicity, employment status and monthly income, due to $p$-value $>0.05$. These findings are further supported by previous studies (Ciechanowski et al., 2001; Dolder et al., 2002; Horne \& Weinman, 1999; Shenolikar et al., 2006; Williams et al., 1998) and these findings do not agree with some literature that found factors (such as gender, educational level and overweight) are significant for medication adherence among T2DM patients(Aljasem et al., 2001; Hauber et al., 2009).

\section{LIMITATION}

While this study complements to the present literature on medication knowledge and adherence among patients with T2DM, the data resulting from the study was partial to one hospital of UMMC in Kuala Lumpur, and limited to the outpatient diabetic clinic via convenience sampling method. However, the sample size of the study was large enough to evaluate the expected differences and association between study variables. Furthermore, another limitation of the study was self-report of medication adherence that might be miscalculated by patients. Conversely, self-reported measure for medication knowledge and adherence demonstrated to have respectable reliability and validity with the ease of presentation. Additional point that might limit the generalization of the study results is the choice of patients who recognize their diabetes medications. Those patients might be considered more knowledgeable than those who did not know, and this may confound the knowledge results.

\section{Implication}

Given the importance of patient' self-management in managing diabetic. This study contributed to the body of knowledge in addressing the issue of medication knowledge and adherence, in particular the relationship between medication knowledge and adherence among 
T2DM patients. The findings of the study have important implication for T2DM patients through giving more of a sense of controlling their treatment so as to be more conscious about what happens to them and the results would be generally better. As more knowledge about $\mathrm{DM}$ in diabetic patients is associated with better medication adherence and better glycemic control. Therefore, through promoting and enhancing patient and clinician awareness that other opportunities such as group education sessions and combinatorial behavioral changes may also produce better outcomes. The importance of these alternatives would preferably increase both patient knowledge and the desire to practice self-management of their medication. Overcoming barriers to self-management will help achieve the goal of the integral outcome of growing patient's quality of life.

However, one of the greatest common challenges that healthcare providers face with a patient with poorly controlled diabetes is to try and figure out if the patient's hyperglycemia is due to non- adherence or is happening despite appropriate medication use. Therefore, Poor medication adherence regimens a republic, contributing to considerable worsening of illness, death and amplified health-care costs. Consequently, physicians should continuously look for poor medication adherence and can improve medication adherence by highlighting the importance of a patient's routine, creating a humble regimen and modifying the regimen to the DM patient's lifestyle. Thus, the findings of the study could direct health care providers on which features of diabetes and its management they should focus their patient's education efforts on. In short, healthcare providers should address patient's beliefs about medications in the hope of improving medication adherence and therapeutic outcome. Additionally, healthcare workers need to assess and educate patients about diabetes mellitus to improve the level of medication adherence and consequently therapeutic outcome.

\section{RECOMMENDATIONS}

Based on the study results, a few recommendations could be made. Firstly, the study findings exposed the need to develop and direct interventions to improve medication knowledge among T2DM patients. In general, continuous education is recommended for all patients and pharmacist intervention is very essential as age and educational level were shown to be the influential factors in this study. Assessment on the levels of medication knowledge amongst patients would be approved from period to period to safeguard patient enhancement and intervention efficiency. Second, there is a need for educational programs to improve the selfmanagement with T2DM, especially we recommend interventions that address the reasons of non-adherence that are addressed in this study in order to increase adherence. Nearly all of such interventions comprise of the pharmacists and physicians improving on the zones of educational patients and medication advising, communication between them and patients, encouraging patients to screen their blood glucose level frequently, simplifying drug regimen with decreasing the number of drug occupied, medication assortment attitude in awareness cost and impossible event effects of the medications.

\section{CONCLUSION}

Diabetes mellitus is one of the non-communicable diseases which are growing very wild in this recent era. Diabetes and linked complications stance a foremost healthcare burden worldwide and recent key challenge to patients, health care systems and national economies. Self-management is one of the most important ways to control hyperglycemia among T2DM. A cross sectional study was carried out to assess the medication knowledge and medication adherence as well as to determine the association between these two variables with the participants' socio-demographic characteristics. A total of 223 patients with T2DM in UMMC were participated in this study. The general findings from the current study showed that there is no correlation between medication knowledge and medication adherence among study participants. As the total number of patients with poor medication knowledge was higher than those with high medication knowledge, it was conversely in terms of medication adherence. Moreover, the last research objective that aimed to determine the association between participants' socio-demographic factors, medication knowledge and medication adherence, was analyzed using chi-square test with the $p$-value $<0.05$. The chi-square test results showed that gender, educational level, employment status, and monthly income were significant factors with medication 
knowledge, while none of the socio-demographic variables was significant in medication adherence due to the $p$-value $>0.05$.

In short, this study gives insight into the numerous factors that affect patient adherence to medication guidelines among Malaysian patients. These factors should be targeted by healthcare professionals. Awareness of these factors will allow healthcare professionals to be more effective in their medication counseling and more self-responsible to regulate and control their blood glucose.

\section{ACKNOWLEDGEMENTS}

We are full of grateful appreciations towards the Medical Ethics Committee of University Malaya Medical Centre for giving ethical approval. Also, we are grateful to Postgraduate Research Grant in the University of Malaya to support and approve grant for conducting this study. The authors would like to thank all lecturers in department of nursing science and all staff in outpatient diabetes clinics in UMMC for their kind support and help throughout the successful completion of the study.

\section{REFERENCES}

Al-Qazaz, H.K., Sulaiman, S.A., Hassali, M.A., Shafie, A.A., Sundram, S., Al-Nuri, R. \& Saleem, F. (2011). Diabetes knowledge, medication adherence and glycemic control among patients with type 2 diabetes. Internationaljournal of clinical pharmacy, 33(6), pp 1028-1035.

Aljasem, L.I., Peyrot, M., Wissow, L. \& Rubin, R.R. (2001). The impact of barriers and self-efficacy on self-care behaviors in type 2 diabetes. The Diabetes Educator, 27(3), pp 393-404.

Burge,S.K, Albright, T. \& RRNeST Investigators (2002). Complementary and alternative medicine use among family practice patients in South Texas. American Journal of Public Health, 92(10), pp 1614-1616.

Chew, B.H., Ismail, M., Lee, P.Y., Taher, S.W., Haniff, J., Mustapha, F.I. \& Bujang, M.A. (2012). Determinants of uncontrolled dyslipidemia among adult type 2 diabetes in Malaysia: The Malaysian Diabetes Registry 2009. Diabetes research and clinical practice, 96(3), pp 339-347.

Ciechanowski, P.S., Katon, W.J., Russo, J.E. \& Walker, E.A. (2001). The patient provider relationship: attachment theory and adherence to treatment in diabetes. American Journal of Psychiatry,158(1), pp 29-35.

Dolder, C.R., Lacro, J.P., Dunn, L.B. \& Jeste, D.V. (2002). Antipsychotic medication adherence: is there a difference between typical and atypical agents? American Journal of Psychiatry, 159(1), pp 103-108.

Danaei, G., Finucane, M.M., Lu, Y., Singh, G.M., Cowan, M.J., Paciorek, C.J., Stevens, G.A., Rao, M., Ali, M.K., Riley, L.M., Robinson, C.A. \& Ezzati, M. (2011). National, regional, and global trends in fasting plasmaglucose and diabetes prevalence since 1980: systematic analysis of health examination surveys and epidemiological studies with 370 country-years and $2 \cdot 7$ million participants. The Lancet, 378(9785), pp 31-40.

Hernandez-Tejada, M.A., Campbell, J.A., Walker, R.J., Smalls, B.L., Davis, K.S. \& Egede, L.E. (2012). Diabetes empowerment, medication adherence and self-care behaviors in adults with type 2 diabetes. Diabetes technology \& therapeutics, 14(7), pp 630-634.

Hauber, A., Mohamed, A., Johnson, F. \& Falvey, H. (2009). Treatment preferences and medication adherence of people with Type 2 diabetes using oral glucose-lowering agents. Diabetic Medicine, 26(4), pp 416-424.

Horne, R. \& Weinman, J. (1999). Patients' beliefs about prescribed medicines and their role in adherence to treatment in chronic physical illness. Journal of psychosomatic research, 47(6), pp 555-567.

Jimmy, B., Jose, J., Al-Hinai, Z.A., Wadair, I.K. \& Al-Amri, G.H. (2014). Adherence to Medications among Type 2 Diabetes Mellitus Patients in Three Districts of Al Dakhliyah Governorate, Oman: A cross-sectional pilot study. 


\section{Sultan Qaboos Universitymedical journal, 14(2), pp e231-e235.}

Kosek, M., Bern, C. \& Guerrant, R.L. (2003). The global burden of diarrhoeal disease, as estimated from studies published between 1992 and 2000. Bulletin of the World Health Organization, 81(3), pp 197-204.

Letchuman, G., Wan Nazaimoon, W., Wan Mohamad, W.B., Chandran, L.R., Tee, G.H., Jamaiyah, H. \& Ahmad Faudzi, Y. (2010). Prevalence of diabetes in the Malaysian national health morbidity survey III 2006. The Medical Journal Malaysia, 65(3), pp 180-186.

Mosher, H.J., Lund, B.C., Kripalani, S. \& Kaboli, P.J. (2012). Association of health literacy with medication knowledge, adherence, and adverse drug events among elderly veterans. Journal of health communication, 17(sup3), pp 241-251.

Mastura, I., Chew, B.H., Lee, P.Y., Cheong, A.T., Ghazali, S.Jamaiyah, H., Alwi, S.A.R.S., Wahyu,T.S. \& Zaiton, A. (2011). Control andtreatment profiles of 70,889 adult type 2 diabetes mellitus patients in Malaysia-across sectional survey in 2009. International Journal of Collaborative Research onInternal Medicine \& Public Health, 3(1), pp 98-113.

Nesto, R.W. (2004). Correlation between cardiovascular disease and diabetes mellitus: current concepts. The Americanjournal of medicine, 116(5), pp 11-22.

O’Kane, M.J., Bunting, B., Copeland, M. \& Coates, V.E. (2008). Efficacy of self monitoring of blood glucose in patients with newly diagnosed type 2 diabetes (ESMON study): randomized controlled trial. British Medical Journal, 336(7654), pp 1174-1177.

Omar, M.S. \& San, K.L. (2014). Diabetes Knowledge and Medication Adherence Among Geriatric Patient with Type 2 Diabetes Mellitus. International Journal of Pharmacy and Pharmaceutical Sciences, 6 (3), pp 103-106.

Shaw, J.E., Sicree, R.A. \& Zimmet, P.Z. (2010). Global estimates of the prevalence of diabetes for 2010 and 2030. Diabetes research and clinical practice, 87(1), pp 4-14.

Sokol, M.C., McGuigan, K.A., Verbrugge, R.R. \& Epstein, R.S. (2005). Impact of medication adherence on hospitalization risk and healthcare cost. Medical care, 43(6), pp 521-530.

Shenolikar, R.A., Balkrishnan, R., Camacho, F.T., Whitmire, J.T. \& Anderson, R.T. (2006). Race and medication adherence in Medicaid enrollees with type-2 diabetes. Journal of the National Medical Association, 98(7), pp 1071-1077.

Sajith, M., Pankaj, M., Pawar, A., Modi, A. \& Sumariya, R. (2014). Medicationadherence to antidiabetic therapy in patients with type 2 diabetes mellitus. International Journal of Pharmacy and Pharmaceutical Sciences, 6, pp 564-570.

Vlasnik, J.J., Aliotta, S.L. \& DeLor, B. (2005). Medication adherence: factors influencing compliance with prescribed medication plans. The Case Manager, 16(2), pp 47-51.

Williams, G.C., Rodin, G.C., Ryan, R.M., Grolnick, W.S. \& Deci, E. L. (1998). Autonomous regulation and longterm medication adherence in adult outpatients. Health Psychology, 17(3), pp 269-276. 